\title{
POLÍTICAS DE ESTADO EN DEMOCRACIA: LA RELACIÓN ESTADO/SOCIEDAD COMO ÁMBITO DE CONSTRUCCIÓN DE LA POLÍTICA*.
}

\section{State policy in democracy: the relationship between State and society as the arena for political construction.}

Melina Guardamagna**

Walter José Cueto***

Recibido: 31 de octubre de 2012. Aprobado: 22 de marzo de 2013.

\section{RESUMEN}

Partiendo de la afirmación que gobernar es generar políticas, iniciamos hace un año, una tarea de aproximación y discusión sobre el concepto de política de Estado, con el objeto de indagar sobre los límites de esta noción, su sentido y alcance, y la posibilidad de encontrar sus atributos específicos en un contexto democrático. En este segundo trabajo ${ }^{1}$, en la búsqueda por continuar dando mayor precisión al concepto, y partiendo de estas primeras precisiones, nos interesa avanzar específicamente en el análisis del proceso de construcción de una política de Estado en democracia y, en este sentido, dar respuesta a los siguientes interrogantes: ¿cuáles son las características de dicho proceso? ¿dónde se ubica el Estado? y ¿cuál es la dinámica de los viejos y nuevos actores que forman parte del mismo y emergen bajo las actuales condiciones sociales? Para ello será necesario discutir la visión estatista e institucionalista tradicional del concepto, y continuar avanzando en la búsqueda de atributos específicos de este tipo de políticas, entendiendo que constituyen objetivos estratégicos en el ámbito de la relación Estado/sociedad.

PALABRAS CLAVES: Estado, sociedad, política.

\footnotetext{
Este trabajo se enmarca dentro del proyecto de investigación 06/F294: "Políticas públicas y calidad de la democracia El caso de la política presupuestaria en Mendoza”. Secretaría de Ciencia, Técnica y Postgrado. Universidad Nacional de Cuyo. Programa de Incentivos 2011/2013.

** Universidad Nacional de Cuyo, Facultad de Ciencias Políticas y Sociales, Centro de Innovación Institucional para la Gobernabilidad Democrática (CEII), Mendoza, Argentina. Correo electrónico: meliguardamagna@gmail.com.

*** Director Universidad Nacional de Cuyo, Facultad de Ciencias Políticas y Sociales, Centro de Innovación Institucional para la Gobernabilidad Democrática (CEII), Mendoza, Argentina. Correo electrónico: wcueto. uncuyo@hotmail.com.
}

$1 \quad$ El primer artículo donde abordamos la temática se denomina: “¿Hay políticas de Estado en la Argentina? Aproximaciones a un concepto." (Cueto y Guardamagna 2012). 


\begin{abstract}
On the basis of the assertion that to govern is to generate policies, last year we began the task of discussing the concept of State policies in order to inquire about the limits of this notion, its meaning and scope, and the possibility of finding its specific attributes in a democratic context. In this second study, in the quest to continue giving greater precision to the concept and on the basis of these first definitions, we are interested in advancing specifically in the analysis of the process of construction of a State policy in democracy and, in this regard, provide answers to the following questions: What are the characteristics of this process? Where is the State? And what is the dynamic of both old and new actors that are part of the same State and emerge under current social conditions? To answer these questions it is necessary to discuss the statist vision and institutionalist traditional of the concept, and continue to move forward in search of specific attributes of these type of policies, comprehending that they constitute strategic objectives in the field of the relationship between the State and society.
\end{abstract}

KEYWORDS: State, society, policy.

\title{
I. POLÍTICAS DE ESTADO EN DEMOCRACIA: LA RELACIÓN ESTADO/SOCIEDAD COMO ÁMBITO DE CONSTRUCCIÓN DE LA POLÍTICA
}

Hace un año, en el marco de nuestra preocupación por el estudio de las políticas de Estado nos preguntábamos si hay políticas de este tipo en América Latina y, especialmente, en Argentina. Como consecuencia iniciamos una primera aproximación al concepto de "políticas de Estado", problematizando la cuestión e indagando básicamente sobre la posibilidad de encontrar atributos específicos y su sentido, en un contexto democrático. En este nuevo trabajo nos proponemos continuar, ya no solo preocupados por el uso del concepto en el ámbito de la política cotidiana, sino también por el modo académico de tratarlo. Pretendemos profundizar el análisis del proceso de construcción de este tipo de políticas en el ámbito de la relación Estado/sociedad; desde la dinámica de los actores que emergen bajo las condiciones socio-políticas actuales. Tratamos de precisar las características y alcance de las políticas de Estado, partiendo de la diversidad de modos y significados con que los autores suelen referirse a las políticas en general. 
En nuestro primer trabajo (Cueto y Guardamagna 2012) sosteníamos que en la política cotidiana se demandan cada vez con mayor frecuencia políticas de Estado; sin embargo, en la mayoría de los casos, lo que en realidad se reclama es la presencia del Estado a través de políticas; lo que sin duda no necesariamente es lo mismo. Es decir, lo que fundamentalmente se le pide a los gobiernos es que gobiernen por políticas, aunque no todas finalmente terminen convirtiéndose en políticas de Estado. Gobernar es, en este sentido, generar políticas, llámense simplemente "políticas", "políticas públicas", "políticas estatales" o "políticas de gobierno"; en principio, se trata de políticas de carácter más inmediato y coyuntural, relacionadas con una gestión gubernamental particular.

Por su parte, la propia sociedad reclama y valora políticas de largo plazo que con frecuencia se sintetizan en la demanda por "políticas de Estado", que se suponen más estables y continuas. Es decir, que percibe en ellas atributos que se sintetizan en ideas de largo plazo, estabilidad, continuidad y consenso. No obstante, una política con un amplio consenso social y político, y con miras de continuidad, no necesariamente se convertirá en una política de Estado. Además, una política no puede ser calificada a priori como "política de Estado"; en todo caso, puede planteársela como una aspiración. Solo el desarrollo del proceso sociopolítico que la envuelva podrá finalmente alentar una posibilidad tal. Por ello una política de Estado no puede pensarse como un programa unilateral, rígido, definido previamente, sino como el resultado complejo y cambiante de la combinación de fuerzas políticas, equilibrios sociales y tendencias históricas y culturales (Natanson 2008).

De acuerdo a estas primeras afirmaciones, una política de Estado es el resultado de un proceso sociopolítico; en consecuencia, ¿cuáles son las características de dicho proceso? y ¿dónde se ubica el Estado en el mismo? Para responder a estas interrogantes es necesario comenzar por discutir el lugar que ocupa el Estado en la definición de las políticas.

Empecemos por reconocer la naturalidad con la que se acepta que únicamente el Estado puede elaborar políticas, premisa que pareciera acompañar siempre implícita o explícitamente al concepto de Estado en la mayoría de sus concepciones e incluso desde el origen del análisis de políticas públicas como subcampo disciplinar. Quizás esta visión también se acompañe con otra idea bastante aceptada de que el Estado es el que define unilateralmente cuándo una política es de Estado y cuando no lo es. Sin embargo, aunque este sea el que lidere los procesos, es importante advertir que las políticas transcurren y se desarrollan en el espacio más complejo y controversial de la relación Estado/sociedad. Es 
decir, que todo concepto de Estado supone asimismo uno de sociedad y es en la tensión de estos dos campos donde emergen las políticas.

Para abordar la relación Estado/sociedad como ámbito de construcción de una política y en especial de una política de Estado es posible entonces distinguir dos perspectivas: una que coloca el acento en la "estatización de la política", en el reconocimiento sustancial de que las políticas se producen y reproducen en el campo específico y exclusivo del Estado, y otra que pone en cuestionamiento esta visión. Esa segunda alternativa, tanto conceptual como de la experiencia, es la de la "desestatización de la política" (Cansino 2008: 171), que sostiene que las políticas igual se producen y reproducen en el campo de la sociedad o por lo menos en un espacio de coproducción activa. En otros términos, se pueden distinguir al menos dos visiones que contextualizan las políticas: una institucionalista, estatista y centralista; y otra, no institucionalista y no estatista que permite dar cuenta de la dinámica de los procesos en los que el Estado no siempre ni necesariamente ocupa un lugar central y hegemónico.

\section{VISIONES EN EL PROCESO DE CONSTRUCCIÓN DE LAS POLÍTICAS EN DEMOCRACIA}

¿Cómo se concibe a las políticas y en particular a las políticas de Estado desde una visión institucionalista y estatista? El concepto de política pública se mueve en un rango de definiciones muy variadas, con énfasis muy distintos, desde opciones de máxima amplitud que prácticamente abarcan toda la vida del Estado, a opciones restringidas a ciertos tipos de acción pública muy específica, que dejan por fuera dimensiones muy importantes, aunque complejas, de la interacción Estado/sociedad (Vieira 2011). No obstante, la mayoría de las definiciones de políticas tienen cuatro elementos coincidentes: gobierno, problemas, objetivos y proceso (Roth 2002: 27); es decir, el actor es uno solo: el gobierno para unos, el Estado para otros, pero uno.

La gran mayoría de los expertos insisten en que solo el Estado puede elaborar políticas. Este supuesto ha estado incorporado de manera general en la mayoría de los argumentos con los que los autores han trabajado el campo disciplinar de las políticas públicas, aun reconociendo que en la base de las mismas pueden haber tradiciones teóricas muy diferentes. La propia definición de "política pública", aunque con diversas connotaciones, siempre termina reconociendo la centralidad del Estado, que es el dueño del juego, lo abre cuando quiere y hasta donde quiere; es el que define la dirección de los procesos y también su extensión. "Las políticas públicas en su esencia están conectadas 
fuertemente al Estado, quien determina cómo son usados los recursos para beneficio de sus ciudadanos" (De Souza 2006: 45).

Sin embargo, no todos los autores son tan categóricos en cuanto a la posición del Estado en el desarrollo de una política. Es el caso de Aguilar cuando define una política pública como:

Conjunto de acciones (secuencia, sistema, ciclo), estructuradas de modo intencional y causal, que se orientan a realizar objetivos considerados de valor para la sociedad o a resolver problemas cuya solución es considerada de interés o beneficio público; acciones cuya intencionalidad y causalidad han sido definidas por la interlocución que ha tenido lugar entre el gobierno y los sectores de la ciudadanía; acciones que han sido decididas por autoridades públicas legítimas; acciones que son ejecutadas por actores gubernamentales o por éstos en asociación con actores sociales (económicos, civiles), y que dan origen o forman un patrón de comportamiento del gobierno y la sociedad (2009: 14).

Es decir, este autor identifica instancias de intervención en paralelo, tanto del Estado como de la sociedad, en la totalidad del proceso.

En consecuencia, las políticas son conjuntos de decisiones definidas e implementadas con la participación y legitimación de autoridades representativas del Estado, junto a representantes de la sociedad, tendientes a mejorar, transformar o solucionar una situación pública problemática o a emprender acciones consideradas útiles para la sociedad. $\mathrm{Y}$ aunque acepta que las acciones puedan ser conjuntas entre autoridades legítimas e instituciones de la sociedad, no considera la opción de políticas solo desde la sociedad y menos aún sin el gobierno. Además, toda política, según este autor, implica de una u otra forma el involucramiento del gobierno o de alguna agencia del Estado, pero no toda acción estatal o gubernamental la reconoce como una política.

Otra visión que pone el acento en el rasgo institucional de las políticas, es la de Subirats, en cuyo modelo de análisis, "las estrategias, las ideas, los intereses y las formas de operar de los actores dependen, esencialmente, de factores derivados de los recursos de que disponen y de su 'posición institucional', acercándose al institucionalismo centrado en los actores" (2008: 25). El modelo propuesto se enmarca, aunque con algunas salvedades, dentro de la generalizada interpretación de las políticas públicas como respuestas institucionales a problemas sociales que llegan a ser reconocidos políticamente como públicos, lo que supone que integran la agenda política. 
Nos situamos en un punto intermedio entre la visión del Estado-ventanilla, neutro y atento a todas las reivindicaciones sociales, y la del Estado "cautivo" y manipulado por un grupo organizado. Desde esta óptica las políticas públicas constituyen una respuesta a un problema público que refleja un problema social (cambiante) que se ha articulado a través de mediadores (por ejemplo, medios de comunicación, nuevos movimientos sociales, partidos políticos y/o grupos de interés) para debatirse posteriormente en el proceso democrático de toma de decisiones (Muller 1990, citado en Subirats et al. 2008: 34).

En esta conceptualización de las políticas públicas, es el marco institucional específico el que pone límites a las acciones de los diferentes actores públicos, parapúblicos y privados, para resolver un problema colectivo que requiere una acción consensuada. Desde esta perspectiva, una política pública se define como:

Una serie de decisiones o de acciones, intencionalmente coherentes, tomadas por diferentes actores, públicos y a veces no públicos -cuyos recursos, nexos institucionales e intereses varían- a fin de resolver de manera puntual un problema políticamente definido como colectivo. Este conjunto de decisiones y acciones da lugar a actos formales, con un grado de obligatoriedad variable, tendientes a modificar la conducta de grupos sociales que, se supone, originaron el problema colectivo a resolver (grupos-objetivo), en el interés de grupos sociales que padecen los efectos negativos del problema en cuestión (beneficiarios finales) (Subirats et al. 2008: 36)².

No obstante, más allá de que las políticas suponen la búsqueda de consensos entre los actores vinculados a una problemática social que requiere una solución colectiva, son los actores públicos quienes tienen la potestad de "reconocer la necesidad de considerar una eventual solución pública en relación a como se ha 'formateado' o tematizado el problema o la situación problemática” (Subirats et al. 2008: 129)³. En definitiva, es la

2 Subirats reconoce tres tipos de actores en el espacio más o menos formal y estructurado donde se desarrolla la política: los de carácter público, es decir, los actores político-administrativos; y los de carácter no público, dentro de los cuales se reconocen, los grupos-objetivo -actores cuyo comportamiento se define políticamente como la causa del problema-; y los beneficiarios finales de la política -actores que sufren los efectos negativos del mencionado problema y cuya situación pretende mejorarse a través de la intervención pública. Se trata, en definitiva, de una clasificación compuesta por tres tipos de actores que constituyen "el triángulo de base" de una política pública (Subirats et al. 2008: 54) que delimita previamente el espacio en el que cada actor interviene y la forma en que lo hace.

3 Este conjunto de decisiones y de acciones sólo podrá considerarse como una política pública en la medida en que quienes las toman y las llevan a cabo lo hagan desde su condición de actores públicos. O dicho de otra manera, es necesario que se trate de actores integrados en el sistema político-administrativo o bien de actores privados 
posición institucional que ocupan los actores lo que los condiciona para la formulación de políticas.

En esta visión, se hace una distinción entre los contenidos sustantivos (¿cómo resolver el problema público?) e institucionales (¿qué actores intervienen, con qué recursos y en base a qué reglas institucionales?) de la política; dimensiones que a su vez presentan complementariedad.

El observador debe estar en posibilidad de constatar, a lo largo de las etapas de una política pública, una concreción de la "sustancia" (contenidos) de la política, así como la consolidación de la red de actores implicados, la constitución de un verdadero "capital institucional" (entramado de actores) formado por reglas específicas y la explotación (iterativa) de todo el "abanico de recursos" disponibles (Subirats et al. 2008: 242).

Por otra parte, la mayoría de estos autores reconocen lo institucional como acciones estatales y no como políticas. Ya lo hemos anticipado: se trata de una perspectiva que restringe el alcance y el ámbito del concepto de política, reconociendo como noción general el de acciones gubernamentales o estatales.

Teniendo como referente el sistema político colombiano -afirma Vieira- podría ilustrar varios ejemplos de acciones gubernamentales o de agencias del Estado que no encuadran en el concepto de políticas públicas; considero incluso que pueden generalizarse a todos los países; por ejemplo, la aprobación de un presupuesto, que es una acción gubernamental que no puede considerarse una política pública, lo mismo que abrir una licitación y hacer un contrato, y así muchas otras, pero tal vez la mayor confusión se genera con los Planes de Desarrollo (en Colombia obligatorios para alcaldes y gobernantes en general según la Ley 152 de 1994), que algunos confunden con políticas públicas, pero que como mucho son sólo lineamientos de política o intenciones por especificar, precisamente, a través de políticas públicas, ese es el ideal del "gobierno por políticas públicas" (Vieira 2011).

Consecuentemente, para que el análisis de cualquier política supere los estrechos márgenes de una mirada institucionalista y la concepción pragmático-estatista tradicional es necesario desandar el camino que las piensa y desarrolla como si fueran parcelas o engranajes propios y excluyentes del Estado. En este sentido cobra real dimensión la observación de Cansino cuando señala que "la política escindida de lo social se vacía

que posean la legitimidad necesaria para decidir o actuar en base a una representación jurídicamente fundada (Subirats et al. 2008: 41). 
de significados fuertes y termina siendo un mero patrón de racionalidad institucional tendiente a disminuir riesgos, conciliar intereses, administrar recursos y asignar legítimamente roles y metas" (Cansino 2008: 139).

Aunque de manera más general, y quizás sin la intención de nuestro recorte, el autor está llamando la atención que una política no es simplemente una secuencia formal de acciones y decisiones que responde a una lógica de causa-efecto, sino que hay un sentido sustantivo que da lo social. Por eso es imprescindible recuperar el horizonte y la contextualidad que le da a las políticas la relación Estado/sociedad, cualquiera sea su naturaleza, característica y magnitud. Es decir, que en el proceso de una política, la sociedad no es solo el campo donde se origina el problema o la destinataria/beneficiaria de la misma; también es coautora de todo el proceso de una política.

Por otro lado, es importante hacer una digresión de peso sobre la perspectiva no institucionalista pero sí estatista que hace bastante tiempo aportaron Oscar Oszlak y Guillermo O'Donnell (1981), que aunque sostienen la centralidad del Estado, lo hacen explícitamente en el contexto de la relación Estado/sociedad.

Ya en la década de 1970, Oszlak y O’Donnell intentaban transitar, a través del estudio de las políticas, hacia una teoría del Estado latinoamericano y en ese sentido no dudaron en utilizar el concepto de "políticas estatales" en lugar del más habitual de "políticas públicas"; aunque pareciera que sin una intencionalidad expresa en la forma que dieron al concepto. A nuestro entender estaban haciendo un significativo giro de resignificación en el sentido del concepto. Quedaba explícito que detrás de la idea de política estaban reposicionando al Estado y no simplemente al gobierno. En este sentido sostenían que:

Las políticas estatales permiten una visión del Estado en acción, desagregado y descongelado como estructura global y puesto en un proceso social en el que se entrecruza complejamente con otras fuerzas sociales y apunta directamente a la reconceptualización del tema del Estado y la sociedad (Oszlak y O’Donnell 2008: 563).

El estudio de las políticas estatales, desde la perspectiva que proponemos-decían nuestros autores- ayuda a desagregar y poner en movimiento a un Estado y a actores (clases, fracciones de clase, organizaciones, grupos, eventualmente individuos) que en otros enfoques aparecen como más global y estáticamente definidos. Y agregan, "el proceso social tejido alrededor del surgimiento, tratamiento y resolución de cuestiones ante las que el Estado y otros actores adoptan políticas, es más dinámico y menos estructural" (Oszlak y O’Donnell 2008: 564). 
Si bien su posición aparece inicialmente como excesivamente terminante en relación a cuándo aparece una cuestión que motive la constitución de una política estatal, al señalar expresamente que "la política estatal es la toma de posición del Estado en torno a una cuestión" (Oszlak y O’Donnell 2008: 569), y en tal sentido pareciera que su centralidad es hegemónica y excluyente, es necesario reconocer también que el proceso previo a la toma de posición del Estado -cuando se construye una cuestión social- es complejo, mucho más abierto y amplio. Por eso precisan:

Nos interesa aprender quién lo reconoció como problemático, cómo se difundió esa visión, quién y sobre la base de qué recursos y estrategias logró convertirlo en cuestión. El período de iniciación puede alertarnos cómo interactúan y se expresan el poder relativo de diversos actores, sus percepciones e ideologías, la naturaleza de sus recursos, su capacidad de movilización, sus alianzas y conflictos y estrategias de acción política (Oszlak y O’Donnell 2008: 568).

En definitiva, una política en la mirada de estos autores se vincula conceptualmente y se adjetiva con la idea de "estatal", y solo es una política en tanto expresa una toma de posición del Estado, confirmando que este aparato -en el contexto de la relación Estado/sociedad- es el que decide y resuelve el problema que motivó la emergencia de una cuestión socialmente problemática. Sin embargo, toda política supone un proceso previo configurador y de iniciación que amplía notablemente la participación, movilización, alianzas, estrategias de acción políticas y los conflictos de una diversidad de actores que involucran en el proceso sus percepciones, su ideología y hasta sus propios recursos. Es decir, aunque es esta una visión estatista, no responde a los cánones institucionalistas y recupera una visión sociopolítica dinámica, que resalta una perspectiva de proceso político, sin considerar a las políticas como simples expresiones de acciones públicas segmentadas. Dicen los autores al respecto:

La política estatal no constituye ni un acto reflejo ni una respuesta aislada, sino más bien un conjunto de iniciativas y respuestas, manifiestas o implícitas, que observadas en un momento histórico y en un contexto determinado, permiten inferir la posición del Estado frente a una cuestión que atañe a sectores significativos de la sociedad (Oszlak y O’Donnell 2008: 569-70).

Incluso, el Estado -diferenciado, complejo, contradictorio- aparece como un actor más en el proceso social desarrollado en torno a una cuestión y su intervención supone "tomar partido", sea por acción u omisión. 
Aunque dichos autores no hablan de políticas de Estado, su conceptualización respalda la dimensión y el alcance que, como venimos señalando, caracteriza a este tipo de políticas. Esta caracterización de las políticas estatales nos permite mirar retrospectivamente una política y ver cómo determinados nudos de problemas se van interconectando en el tiempo, permitiendo su continuidad y acercándonos a la idea de políticas de Estado. "En el caso de las políticas de Estado, que trascienden períodos de gobierno, el énfasis está puesto, más que en el contenido, en el proceso, es decir, habría una visión más instrumental de la política" (Cueto y Guardamagna 2012: 16).

Es significativo el proceso de constitución de una cuestión socialmente problematizada y coincidimos con Oszlak y O'Donnell que esas cuestiones se constituyen en una dinámica muy fuerte entre el Estado y la sociedad. Sin embargo, hay una segunda instancia de este proceso en el cual los autores reconocen la centralidad absoluta del Estado en el momento en el que toma posición frente a la resolución de una cuestión. Este último argumento no lo compartimos porque la exclusividad estatal en la resolución de los problemas no se verifica en el proceso de la política cotidiana donde siempre algún sector de la sociedad -aunque no sea toda-incide en la definición. Es decir, también en la instancia de resolución de un problema está presente la sociedad o como mínimo algún sector.

Por otro lado, una cuestión puede volverse socialmente problematizada mucho antes que el Estado tome posición en el proceso de construcción de la misma. Por ello es importante entender cómo se da la vinculación Estado/sociedad y comprender que en estos procesos históricos el Estado no siempre ocupa el lugar central. Hay cuestiones socialmente problemáticas, que en términos de temporalidad, algún actor social se adelanta al Estado y al hacerlo comienza a desarrollar una política. Es decir, aunque el Estado en algún momento toma posición frente a la cuestión no necesariamente es el que la inicia, el que la impulsa y aun el único que la resuelve.

Más allá de lo cual, y retomando algunas de las visiones que explican el proceso de construcción de las políticas, lo cierto es que en el debate sobre si el Estado es una "entidad" o una "relación", el institucionalismo tradicional parece adscribir claramente al primer término y en tal sentido su idea de Estado está asociada a la de entidad o aparato, mientras que la posición que Oszlak y O'Donnell pareciera adscribirlos concibiendo al Estado como una relación (Alford y Friedland 1991: 36), mirada que nos interesa recuperar.

Frente a estas visiones institucionalistas, estatistas y centralistas, y sus diversas perspectivas, que -casi como un implícito- acompañan el tratamiento de las políticas y 
sin desconocer la relevancia de la posición del Estado en sus procesos, nos interesa resaltar en el debate académico, político y social la necesidad de privilegiar en nuestra democracia la relación Estado/sociedad como categoría central, inescindible y una perspectiva que privilegie una mirada histórico-estructural, reposicionando el lugar del Estado y relativizando los excesos de institucionalismo en el análisis.

En consecuencia nos preguntamos: ¿cómo se concibe a las políticas y en particular a las políticas de Estado desde una visión no institucionalista y no estatista? Precisamente, en esta dirección, ha venido cobrando gran importancia en los últimos años una visión contraria a la institucional que postula la desestatización de la política en virtud de las propias transformaciones que ha experimentado la cuestión social (Cansino 2008: 171)4.

La realidad de nuestra democracia plantea de modo creciente la necesidad de construir una visión o un enfoque alternativo para el análisis y comprensión de las políticas que ponga en caja los institucionalismos que con frecuencia concluyen en la mera formalización de los procesos y en la priorización de las regularidades o rutinas que de ellos pueda inferirse. Pero además es necesario pensar en una concepción que desestatice el estudio de la política y las políticas. No es un reclamo que ataque el espacio propio del Estado, sino su absolutidad y excesiva centralidad; cuando desconoce en teoría los espacios que en la práctica cotidiana gana la sociedad, tratados como simples situaciones de excepción.

Además, la emergencia de una importante diversidad de fenómenos políticos incorpora densidad y complejidad a la dinámica estatal y en especial a la producción de políticas. Tal como lo explica Cansino:

El avance de la sociedad ante los experimentos institucionales neocorporativos o neoliberales sufridos por el Estado están marcando y dejando en evidencia algunas cuestiones de relieve: a) la crisis de los partidos políticos en las democracias modernas, los cuales tienen cada vez más dificultades para representar y agregar intereses sociales, pues responden cada vez más a las utilidades de sus elites internas; b) el imperativo de redefinir los alcances y los límites de las esferas del Estado y la sociedad, a la luz de la emergencia de nuevos actores y movimientos sociales; y c) la pérdida de eficacia de las tradicionales fórmulas de gestión económica y social de orden corporativo y clientelar

4 En esta tradición confluyen, según César Cansino, autores como Hannah Arendt (1958 y 1971), Cornelius Castoriadis (1975), Claude Lefort (1983 y 1986) y más recientemente Helmut Dubiel (1989) y Agapito Maestre (1994 y 2000). 
y el cuestionamiento público del universo de los políticos por motivos de corrupción y nepotismo (2008: 140).

Lo cierto es que no solo hay diversas lecturas sobre el alcance del concepto de sociedad, sino también sobre la relación Estado/sociedad y cada una de esas lecturas supone diversidad de respuestas y dilemas que demandan definición.

Se trata, en definitiva, de una visión "en proceso de construcción" que se enmarca en la idea de que "un Estado y una sociedad democráticos son incompatibles con poderes invisibles, con instituciones políticas elitistas u oligárquicas y con decisiones secretas y por la otra, con relaciones sociales y organizaciones que puedan distorsionar los resultados democráticos" (Cansino 2008: 150); perspectiva desde la cual, generar políticas de Estado requiere de un espacio o un campo político donde Estado y sociedad se piensen entre y a sí mismos; para ello los gobiernos deben alentar una institucionalidad que facilite la circulación de los conflictos y del debate público.

\section{LA DINÁMICA DE LOS ACTORES}

Comprender la relación Estado/sociedad como ámbito de construcción de una política y en especial de una política de Estado bajo la dinámica de los viejos y nuevos actores que emergen en la escena política y social actual, supone estar atentos al rol que estos juegan en dicha relación.

En las sociedades actuales los actores se diversifican buscando integración más que consenso (Cansino 2008: 180) y nuevas formas de coordinación (Lechner 1997: 151); sumado a lo cual:

La democratización ofrece condiciones favorables para la movilización de los recursos de las organizaciones de la sociedad civil y oportunidades políticas propicias para su intervención. El levantamiento de las restricciones legales a la acción política reduce los costos de la acción colectiva. La institucionalización de la competencia política y la apertura de la esfera pública valorizan las capacidades de movilización, producción de información y argumentación de las organizaciones de la sociedad civil (Goldstone 2004, citado en Leiras 2007: 35). 
La influencia de estos nuevos actores que emergen en la escena política actual está asociada, además, a la cada vez mayor diversidad de temas y problemas que requieren solución y se convierten en objeto de políticas.

La expansión temática reduce la probabilidad de que los funcionarios públicos (electos o designados) dispongan de información en cantidad y calidad suficientes para tratarlos. Este déficit de información abre la puerta a la influencia de individuos y organizaciones que tienen los incentivos políticos o la competencia profesional para producirla. En la medida en que la cantidad y complejidad de los temas de incumbencia estatal aumenten, esperaríamos que aumente también la cantidad de las asociaciones de la sociedad civil destinadas al tratamiento especializado de temas específicos de política (Braun et al. 2007, citado en Leiras 2007: 37).

En este contexto, tal como sostiene Leiras, es fundamental entender la influencia de estas mediaciones para caracterizar las relaciones entre el Estado y la sociedad civil en contextos específicos (2007: 38).

Además, es necesario señalar que, como afirma Oszlak, al esbozar un marco conceptual que contempla los principales aspectos a considerar en el análisis de los procesos de implementación participativa de políticas públicas, existen múltiples circunstancias y modalidades de participación ciudadana, que, desde el ámbito teórico, no han llegado aún a constituirse en categorías analíticas que permitirían formular una teoría general aceptable sobre el tema (2009: 5). Habiendo hecho esta salvedad, es importante reconocer los diversos roles que las organizaciones de la sociedad civil (OSC) juegan en su relación con los poderes estatales.

La mayoría de los análisis se concentran en dos roles: el de promotoras de temas y evaluadoras de la gestión pública (advocates) y el de socias territoriales en la implementación de programas. Otros dos papeles, igualmente relevantes, merecen consideración. Uno, citado con alguna frecuencia, es el de promotoras de innovaciones. La escala pequeña y la flexibilidad permiten que las OSC ensayen estrategias de intervención no probadas a muy bajo costo. Las innovaciones exitosas de parte de las organizaciones de la sociedad civil enriquecen la trama social y pueden servir de modelo o de instancia de validación para la innovación en política pública. Menos frecuentemente se reconoce (quizás porque ocurre frecuentemente) el rol que las organizaciones de la sociedad civil pueden cumplir como facilitadoras de la negociación entre funcionarios públicos. Esto puede ocurrir tanto entre distintos grupos legislativos, entre legislado- 
res y funcionarios del Ejecutivo o entre funcionarios de distintos niveles de gobierno (por ejemplo, nacionales y provinciales) (Leiras 2007: 42).

Lo cierto es que, en relación a los diversos ámbitos y modalidades de participación ciudadana, los procesos de descentralización por los que atravesó el Estado en la Argentina, principalmente durante la década de 1990, permitieron, con mayor énfasis en el plano local y municipal, una intervención más inmediata y directa de los ciudadanos en el diseño de políticas públicas y en el control de la gestión (Oszlak 2009: 5), ampliando los ámbitos de participación de actores sociales que hasta entonces no tenían la posibilidad de ser generadores de políticas.

Esto supone que las cuestiones socialmente problematizadas involucran actores que pertenecen a la sociedad civil y que también tienen la capacidad de definir políticas. Oszlak y O’Donnell lo reconocían:

Otros actores, además del Estado, también toman posición frente a cuestiones que los afectan, adoptando políticas cuyas consecuencias pueden incluir considerablemente el proceso de resolución de las cuestiones y las futuras tomas de posición sobre las mismas [...]. Cada práctica, cada toma de posición, refleja una determinada estrategia de acción cuyas premisas dependen del volumen de recursos y apoyos que el actor pueda movilizar y de sus expectativas acerca del comportamiento de los otros actores afectados por la cuestión (2008: 115).

En los hechos, la participación ciudadana se manifiesta, de acuerdo a Oszlak, en los procesos de formación de las políticas públicas; en la ejecución de las respectivas decisiones y en la evaluación o control de la gestión pública (2009: 11). En definitiva, se trata de actores que se constituyen como tales a partir de las cuestiones que requieren solución y en función de las cuales definen su modalidad de involucramiento en las mismas. Sentido en el cual, el conjunto de políticas -cualquiera sea los actores que las impulsan- se entrelazan en un complejo proceso social que hace difícil establecer la proporción del cambio social atribuible a los actores y a las políticas que impulsan.

En otros términos, se trata de un contexto en el que las iniciativas ciudadanas son cada vez más frecuentes y el Estado tiende a ser rebasado permanentemente, mientras la sociedad civil es considerada "el lugar donde los ciudadanos, en condiciones mínimas de igualdad y libertad, cuestionan y enfrentan cualquier norma o decisión que no haya tenido su origen o rectificación en ellos mismos" (Cansino 2008: 171), visión desde la cual la sociedad civil adquiere mayores niveles de autonomía frente al Estado. En este sentido, y 
tal como sostiene Cansino, "la democracia en las sociedades modernas debe dar cobertura teórica al conjunto de iniciativas ciudadanas, movimientos sociales y demás acciones que como tales llenan de nuevos contenidos simbólicos al poder político" (2008: 173). Por lo tanto, si entendemos que gobernar es generar políticas, entonces el análisis de políticas también debe incorporar a la sociedad civil y a sus diversas manifestaciones como actores con autonomía, capaces de definir agenda e impulsar políticas, e incluso sostener políticas de Estado en el tiempo.

Desde la teoría, decíamos que existen diversas lecturas sobre el alcance de la relación Estado/sociedad, algunas de las cuales relativizan la centralidad del Estado en la elaboración de las políticas. Una de ellas, es el enfoque que estudia la dinámica de los actores a través de la coordinación mediante redes.

En el escenario de las complejas sociedades actuales el Estado ha dejado de ser el articulador por excelencia de la vida social, debido a que la coordinación política centralizada por parte del Estado es insuficiente para responder a las múltiples demandas que surgen de sociedades que transitan procesos de reestructuración. La coordinación jerárquica por parte del Estado enfrenta serios obstáculos. "La centralidad del Estado se encuentra cuestionada por la nueva complejidad de la vida social, por el auge de múltiples actores socioeconómicos con capacidad de presionar y 'colonizar' la intervención estatal" (Lechner 1997: 148). De esta forma, mientras el Estado deja de ser la instancia privilegiada de coordinación social, la sociedad civil se encuentra cada vez más fortalecida y altamente diversificada, lo cual genera nuevos procesos de negociación y concertación entre Estado y sociedad, donde la política reaparece en un lugar prioritario, facilitando la "elaboración de consensos acerca de las normas fundamentales y de acuerdos sectoriales" (Lechner 1997: 151).

En este contexto se inserta el reciente auge, sostiene Lechner, de la coordinación mediante redes. Se trata de una "coordinación horizontal entre diversos actores interesados en un mismo asunto con el fin de negociar y acordar una solución” (1997: 155). Para realizar estas afirmaciones, el autor se basa en el trabajo de Dirk Messner, quien enuncia las características propias de una red, dentro de las cuales nos interesa destacar que: una red combina la comunicación vertical y la horizontal; vincula diferentes organizaciones, estableciendo una interacción entre sus representantes; es política cuando reúne actores estatales con actores económicos y sociales; hay independencia recíproca entre sus participantes, ya que ninguno de ellos tiene, por sí solo, todos los recursos necesarios para resolver el problema y depende, por lo tanto, de la cooperación de los demás; y su obje- 
tivo es el de formular y llevar a cabo decisiones colectivas en torno a determinado tema compartido (1995: 211, citado en Lechner 1997: 155-56).

Una premisa básica de este tipo de coordinación es el equilibrio entre Estado y sociedad. Es la combinación de una sociedad fuerte y un Estado fuerte lo que da lugar a las redes políticas como combinación de regulación jerárquica y coordinación horizontal (Lechner 1997: 157). Sin embargo, la coordinación mediante redes enfrenta serios riesgos. Uno de ellos es que no asegura el carácter democrático de la coordinación, dado que no se encuentra intrínsecamente vinculada con las instituciones democráticas clásicas; por lo cual, "sólo el Estado parece estar en condiciones de asegurar la dimensión representativa de la coordinación; así como cierta capacidad de conducción política, aspectos que remiten a la democracia" (Lechner 1997: 161). No obstante, lo interesante de este tipo de enfoques es que nos permiten cuestionar, desde la teoría, la centralidad absoluta del Estado en la formulación de las políticas y, al mismo tiempo, nos ayudan a comprender la dinámica que los actores no estatales juegan en estos procesos.

\section{LAS POLÍTICAS DE ESTADO COMO OBJETIVOS ESTRATÉGICOS EN EL ÁMBITO DE LA RELACIÓN ESTADO/SOCIEDAD}

Las políticas de Estado, que como ya afirmamos no pueden ser pensadas fuera de la relación Estado/sociedad, constituyen la manifestación más significativa de la capacidad de conducción de un Estado, forman parte de las estrategias centrales de un país y se sostienen más allá del color político-ideológico del gobierno. Trascienden la temporalidad de una gestión, la resolución de problemas de coyuntura y aun los de mediano alcance para tratar de definir criterios que resuelvan problemas de nivel estructural. Para ello requieren que desde el nivel gubernamental se establezca y garantice la construcción institucional que consolide el largo aliento de varias de estas medidas, que a priori no pueden calificarse como políticas de Estado.

Una "política de Estado" hace referencia a un tipo particular de política, distinta de una política de gobierno, de la cual, sin embargo, no existe una conceptualización consensuada que permita precisar en el lenguaje político y académico cuándo una política es de Estado, identificando sus atributos operativos y tangibles. No se trata de una cuestión teórica en sí, sino de un problema práctico, por lo cual es necesario que se aporte en la búsqueda de rigor conceptual que ayude a desarrollar un lenguaje con bajo nivel de equivocidad y que contribuya fundamentalmente para que los actores políticos y sociales se entiendan. 
En consecuencia, es necesario distinguir una "política de gobierno" de una "política de Estado". Una política de gobierno es aquella que se circunscribe a una gestión en particular y responde a los intereses y criterios de esta. Por lo tanto, dura mientras esté vigente el gobierno que la concibió. Es decir, son políticas que normalmente no tienen continuidad más allá del propio gobierno que la formuló y la sostuvo. Tampoco busca consensos extendidos, sino que refleja el pensamiento de la mayoría que durante un período determinado sustenta a ese gobierno. Es más, una política de gobierno no tiene por qué tener continuidad con políticas anteriores, no necesariamente tiene consenso ni lo busca, porque la raíz ideológica que diferencia la nueva política con la anterior, es la misma que diferencia al gobierno de la oposición. Consecuentemente, a un gobierno no se le puede exigir que formule políticas de Estado porque esa no es su competencia. Lo que no puede dejar de hacer es gobernar por políticas y por lo tanto, proponerlas, discutirlas, tratar que prevalezcan sus criterios, implementarlas, ejecutarlas y aplicarlas.

Una política de Estado, en cambio, construye cursos complejos que influirán en la vida del Estado y de toda la sociedad. Supone una determinada modalidad de intervención estatal en relación a una cuestión que concita la atención, interés o movilización de todas las organizaciones de la sociedad. Esto es así porque el Estado, a través de sus políticas suele encarnar valores, algunos de los cuales son vertebrales para el desarrollo de la sociedad, en torno a los que se formulan políticas, que más allá del componente ideológico de cada gobierno, perduran en el tiempo, convirtiéndose en verdaderas políticas de Estado.

En otros términos, una política de Estado responde a intereses más generalizados, valores que son menos cuestionables por la mayoría de la sociedad y que además son de suma importancia para el desarrollo del país, aunque no necesariamente exista unanimidad sobre la misma. Muchas políticas se implementan con el desacuerdo de las fuerzas políticas de la oposición o de determinados sectores de la sociedad y sin embargo, a largo plazo, adquieren continuidad y consenso por su relevancia. Esto significa que varía el horizonte temporal de ambos tipos de políticas.

En este sentido, este tipo de políticas se aproximarían a lo que Matus denominó, diferenciándola de la planificación estratégica situacional, "la gran estrategia". Se trata de un tipo de enfoque trascendental que, a diferencia de los planes definidos para un período de gobierno, guía la planificación de un país a largo plazo.

El análisis de gran estrategia es más científico, más independiente de la lucha política contingente y debe realizarse ni muy lejos ni muy cerca del gobierno. Es una explora- 
ción del futuro muy compleja, que requiere gran dominio en las fronteras de las ciencias y alta imaginación y creatividad. Es un tipo de enfoque trascendental: un país que no tiene gran estrategia es necesariamente un país imitador (Matus 2006: 27).

La definición de Matus nos acerca a una de las dos miradas distintas pero complementarias desde las cuales podemos analizar las políticas de Estado, cuando el análisis se orienta a los contenidos de la política, acercándonos al plano del deber ser y a una visión prospectiva, propia del concepto de planificación ${ }^{5}$. La otra mirada, en cambio, se aproxima al concepto de políticas de Estado desde el plano instrumental, para referirnos preferentemente a políticas consolidadas históricamente, y se trata de una visión retrospectiva de la política.

Frente a estas dos perspectivas de análisis, no acordamos con la distinción conceptual propuesta por Claudia Bernazza al comparar "proyectos nacionales" con "políticas de Estado", y menos aún, con la definición que la autora propone de este tipo de políticas atadas al enfoque institucionalista. El objetivo de su trabajo “ ¿Proyectos nacionales o políticas de Estado? Aportes al lenguaje de la política" es "develar los diferentes enfoques que operan detrás de las palabras. Un enfoque naturalista-institucionalista parecería estar jugando en oposición a un enfoque constructivista-situado" (2011: 4), por el cual declara su preferencia. De acuerdo a Bernazza,

un Proyecto Nacional, en tanto propuesta de futuro al que se accedería a través del logro de objetivos situados en un campo ideológico particular, parece contraponerse en el discurso público con las políticas de Estado, presentadas como lo armonioso y permanente (2011:4).

Por lo tanto, mientras "los Proyectos de Nación son un conflicto en sí mismos”, el concepto de política de Estado, "al no debatir el orden establecido ni proponer cambios, se asocia a la ausencia de conflicto" (Bernazza 2011:3).

El problema radica, de acuerdo a la autora, en la traducción e interpretación que, desde la "academia", se ha dado al término de políticas públicas. A partir de una traducción no exenta de ideología, el estudio de las políticas públicas se centró en la acción administrativa de los gobiernos, dejando para otras ciencias o líneas de investigación el

5 "Planificar significa pensar antes de actuar, pensar con método, de manera sistemática; explicar posibilidades y analizar sus ventajas y desventajas, proponerse objetivos, proyectarse hacia el futuro, porque lo que puede o no ocurrir mañana se decide si mis acciones de hoy son eficaces o ineficientes. La planificación es la herramienta para pensar y crear el futuro" (Matus 2006: 18). 
rastreo de la contienda política o electoral que les da origen. Este estudio pareciera centrarse en la acción política que acontece una vez superados los conflictos y dirimidas las cuestiones del poder. En este escenario, ya están definidos los vencedores y los vencidos. La política pública, como producto del sistema político, se desentiende de las cualidades del orden establecido y de la validez de ese orden. El concepto política de Estado está íntimamente ligado al concepto anterior. Y agrega Bernazza, "esta ilusión de estabilidad y permanencia es la que vuelve eficaz el concepto política de Estado” (2011: 3).

Atar el análisis de las políticas, como lo hace Bernazza, a una única mirada supone el riesgo de desconocer que las teorías son construcciones, visiones de la realidad, que así como sostienen, también cuestionan las posturas dominantes. Tal como ya advertimos al principio de este trabajo, existen diversos abordajes al análisis de políticas públicas. El más frecuente es el institucionalista, estatista y centralista, pero no es el único. Sumado a lo cual creemos que es un error desconocer el carácter conflictivo de las políticas de Estado. De hecho, uno de los atributos que caracteriza a este tipo específico de política, es su carácter conflictual. Aunque lo que se vea a simple vista sean los acuerdos y consensos, nada de esto puede ser concebido sin la conflictividad de base que se produce y reproduce en el ámbito de las políticas. En otros términos, las políticas se construyen en un proceso de naturaleza conflictiva en el cual hay momentos en los que la lucha y el disenso dan paso a acuerdos, a instancias que ayudan a generar puntos de encuentro o por lo menos, a aceptar que alguien finalmente logra imponer su visión y eso temporalmente genera un orden. Además, su carácter controversial no solo afecta a las políticas en sus procesos, sino también en sus contenidos.

El conflicto entonces es un atributo común o un prerrequisito para cualquier política, es decir, una política supone siempre ambas dimensiones: conflicto y consenso. Es por ello que la continuidad y el consenso, por sí mismos, son claramente insuficientes para caracterizar una política de Estado. Privilegiar el consenso y el tiempo es solo una visión simplificada, superficial y quizás hasta imprecisa; sin embargo, la continuidad y el consenso pueden alcanzar el carácter de atributo específico de una política de Estado cuando se arraigan sobre la base y como resultado de un proceso extenso, controversial y cambiante. En este sentido, las políticas de Estado son una realidad compleja y dinámica, y más aún en democracia, cuando los acuerdos son el resultado de los constantes debates entre las fuerzas políticas y los actores sociales.

Más allá de desconocer el rol que el conflicto juega en la configuración de las políticas de Estado, atribuyéndoselo a los proyectos nacionales, Bernazza termina, de manera un tanto contradictoria, afirmando que: 
Un Proyecto Nacional (provincial, regional, local) y las políticas de Estado son dos conceptos en diálogo. Sin proyecto, sin tensiones alrededor de diferentes proyectos, sin conflictos y superación de las diferencias, sin acuerdos, sin recorridos históricos, no hay políticas de Estado. Una política de Estado puede visualizarse una vez consolidada, es casi un "darse cuenta" de la estabilización de acuerdos alcanzados luego de arduas negociaciones, o luego de la derrota de alguno de los sectores o proyectos en pugna, generalmente el más débil. Una política de Estado es un producto al que hay que acercarse críticamente, porque lo instituido suele responder a sectores dominantes (2011:4).

\section{CONCLUSIONES}

En la necesidad por avanzar en la búsqueda de atributos específicos que nos ayuden a delimitar con mayor precisión el concepto de políticas de Estado, no creemos que se trate simplemente de una cuestión de enfoques ${ }^{6}$, sino más bien de entender que, frente a las dos miradas distintas pero complementarias desde las cuales podemos analizar las políticas de Estado, al trascender períodos de gobierno, el énfasis está puesto, más que en el contenido, en el proceso, lo que significa que se privilegia una visión más instrumental de la política. En este tipo de políticas, es probable que se generen conflictos y diferencias ideológicas entre el gobierno y la oposición o con el partido que estuvo con anterioridad en el gobierno y aun con diferentes sectores de la sociedad. Es decir, lo que permite configurar la política como política de Estado es el proceso de conflictividad que desarrolla el mismo. De hecho, puede suceder que un gobierno entre en conflicto con una política de Estado, pero también que gobiernos distanciados ideológicamente continúen la misma política de Estado.

En todo caso, el atributo específico de una política de Estado es algún nivel de conjunción entre conflicto, continuidad y consenso, aunque esta característica general no siempre se concrete del mismo modo en la realidad contingente, especialmente con referencia a la continuidad y el consenso, que no siempre parecen conjugarse al mismo tiempo. En determinadas circunstancias puede prevalecer de modo unilateral la continuidad y en otros

6 Bernazza sostiene que "desde un enfoque institucionalista, la política de Estado está vinculada a cuestiones sobre las que parecería haber un acuerdo generalizado (mayor educación, combate de la pobreza, mayores niveles de seguridad), sin poner en juego las causas que originaron lo que se pretende cambiar ni los métodos para producir el cambio. Este enfoque le otorga al gobierno el papel de lo dinámico y coyuntural, por lo que la propuesta de Proyectos Nacionales por parte de éstos es, cuanto menos, poco confiable. Un enfoque constructivista, en cambio, reconoce la incidencia de los gobiernos, y por lo tanto de lo dinámico y coyuntural, en la producción de idearios y políticas de largo aliento" (2011: 4). 
casos el consenso, incluso cuando este último se genera para provocar rupturas o cambios en el curso seguido hasta ese momento. Tampoco es necesario que la política de Estado se dé ininterrumpidamente; una misma política puede plantearse como política de Estado en un período y como política de gobierno en otro, donde no prime ninguno de los dos atributos y en consecuencia la política sea simplemente una política de un gobierno.

En definitiva, comprendemos y acordamos con Bernazza que se trata de conceptos complementarios, pero esto dependerá del lugar donde se ubique la mirada de quien los estudia. Si uno mira al Estado en términos prospectivos, lo hace en términos de planificación; es decir, constituye una mirada asociada a los planes que se proponen para el Estado en cada momento. Si en cambio se mira para atrás, retrospectivamente, lo que se ve son procesos históricos en torno a determinadas cuestiones, lugar desde el cual podemos hablar o no de políticas de Estado. Y esto es así porque las políticas de Estado son el resultado de un proceso histórico, en torno a temas que son centrales en la relación Estado/sociedad y como tal constituyen visiones estratégicas. En este sentido es imposible confundir conceptualmente política de Estado con política neoliberal, es sencillamente inexplicable; de manera análoga a pretender generar una política de Estado por el simple acto de la voluntad política: esa voluntad tendrá primero que pasar el tamiz de una construcción histórica.

\section{REFERENCIAS}

1. Aguilar, Luis. 2009. "Marco para el análisis de las políticas públicas." Pp. 9-45 en Política pública y democracia en América Latina: del análisis a la implementación, coordinado por F. Mariñez y V. Garza. México: Porrúa.

2. Alford, Robert y Roger Friedland. 1991. Los poderes de la teoría. Capitalismo, Estado y democracia. Argentina: Manantial.

3. Bernazza, Claudia. 2011. "Proyectos nacionales o políticas de Estado. Aportes al lenguaje de la política." Revista Reseñas y Debates 65: 1-4.

4. Cansino, César. 2008. La muerte de la ciencia política. Buenos Aires: Sudamericana.

5. Cueto, Walter y Melina Guardamagna. 2012. “ ¿Hay políticas de Estado en la Argentina? Aproximaciones a un concepto." Revista Documentos y Aportes en Administración Pública y Gestión Estatal (DAAPGE) 18: 7-26.

6. De Souza, Celina. 2006. "Políticas públicas: uma revisao da literatura." Sociologías 16: 20-45. 
7. Lechner, Norbert. 1997. “Tres formas de coordinación social. Un esquema." Revista de la CEPAL 61: 143-63.

8. Leiras, Marcelo. 2007. "Relaciones entre Estado y sociedad civil en la Argentina: un marco de análisis." Pp. 11-43 en Construyendo confianza: hacia un nuevo vínculo entre Estado y sociedad civil, volumen I, editado por Fundación CIPPEC. Buenos Aires: Fundación CIPPEC, Subsecretaría para la Reforma Institucional y Fortalecimiento de la Democracia. Jefatura de Gabinete de Ministros, Presidencia de la Nación.

9. Matus, Carlos. 2006. Planificar para gobernar: el método PES. Buenos Aires: Universidad Nacional de la Matanza.

10. -----. 2007. Los tres cinturones del gobierno. Buenos Aires: Universidad Nacional de la Matanza.

11. Natanson, José. 2008. “¿Qué es, en realidad, una política de Estado?” Página 12, 29 de junio. Consultado el 5 de mayo de 2011 (http://www.pagina12.com.ar/diario/elpais/1-106918-2008-06-29.html)

12. Oszlak, Oscar. 2009. "Implementación participativa de políticas públicas: aportes a la construcción de un marco analítico." Pp. 9-48, en Construyendo confianza. Hacia un nuevo vínculo entre Estado y sociedad civil, volumen II, editado por Fundación CIPPEC. Buenos Aires: Fundación CIPPEC y Subsecretaría para la Reforma Institucional y Fortalecimiento de la Democracia, Jefatura de Gabinete de Ministros, Presidencia de la Nación.

13. Oszlak, Oscar y Guillermo O’Donnell. 1981. "Estado y políticas estatales en América Latina: hacia una estrategia de investigación." Centro de Estudios de Estado y Sociedad (CEDES). Documento G.E. CLACSO. Vol. 4. Versión utilizada en Lecturas sobre el Estado y las políticas públicas. 2008. Retomando el debate de ayer para fortalecer el actual. Buenos Aires: Jefatura de Gabinete de Ministros. Presidencia de la Nación.

14. Roth, André-Noel. 2002. Políticas públicas: formulación, implementación y evaluación. Bogotá: Aurora.

15. Subirats, Joan, Peter Knoepfel, Corinne Larrue y Frederic Varonn. 2008. Análisis y gestión de políticas públicas. Barcelona: Ariel.

16. Vieira, Juan. 2011. El estudio de las políticas públicas. Medellín. Consultado el 12 de mayo de 2012 (http://politicas-y-publicas.blogspot.com/). 